\title{
A Study on Karyotype of the Asian Leopard Cat, Prionailu- rus bengalensis (Carnivora, Felidae) by Conventional Staining, G-banding and High-resolution Technique
}

\author{
Puntivar Keawmad, Alongkoad Tanomtong* and Sumpars Khunsook \\ Genetics Program, Department of Biology, Faculty of Science, Khon Kaen University, \\ Muang, Khon Kaen, 40002, Thailand
}

Received January 9, 2007; accepted January 31, 2007

\begin{abstract}
Summary A karyotypic study of the Asian leopard cat (Prionailurus bengalensis) in Thailand has been made. Blood samples were taken from 2 males and 2 females. After the standard whole blood lymphocyte culture in the presence of colchicine, the metaphase spreads were performed on microscopic slides and air-dried. Conventional staining, G-banding and high-resolution techniques were applied to stain the chromosomes. The results showed that $2 n$ of the Asian leopard cat was 38 , and the fundamental number (NF) was 74 in male and female. There are 6 autosome types: A type has 4 large and 2 medium submetacentric chromosomes, B type has 6 large and 2 medium acrocentric chromosomes, $\mathrm{C}$ type has 4 large metacentric chromosomes, D type has 8 small submetacentric chromosomes, E type has 8 small metacentric chromosomes and $\mathrm{F}$ type are 2 small telocentric chromosomes. A pair of the short arm of chromosome E1 (chromosome pairs 14) showed a clearly observable satellite chromosomes. The $\mathrm{X}$ chromosome was medium submetacentric chromosome and the $\mathrm{Y}$ chromosome was the smallest metacentric chromosome. From the G-banding and high-resolution techniques, the number of bands and locations in the Asian leopard cat was 183 and 236 respectively, and each chromosome pair could be clearly differentiated. We found that chromosomes A1, A2, A3, B3, B4, C1, C2, D3, D4, E1, E3, F1 and X chromosome patterns were according to the domestic cat (Felis catus) chromosomes. Chromosomes B1, B2, D2 and E2 are similar to those of the domestic cat. These results show the evolutionary relationship between the Asian leopard cat and the domestic cat. The karyotype formula for the male and female Asian leopard cat is as follows:
\end{abstract}

$$
2 n(38)=\mathrm{L}_{4}{ }^{\mathrm{m}}+\mathrm{L}_{4}{ }^{\mathrm{sm}}+\mathrm{L}_{6}{ }^{\mathrm{a}}+\mathrm{M}_{2}{ }^{\mathrm{sm}}+\mathrm{M}_{2}{ }^{\mathrm{a}}+\mathrm{S}_{8}{ }^{\mathrm{m}}+\mathrm{S}_{8}{ }^{\mathrm{sm}}+\mathrm{S}_{\mathrm{t}}{ }^{2}+\text { Sex-chromosome }
$$

Key words Karyotype, Asian leopard cat (Prionailurus bengalensis), Conventional staining.

Over the world, animals in the family Felidae are separated into 18 genera and 36 species. Six genera and 9 species are found in Thailand (Wilson and Cole 2000). These are marbled cat (Pardofelis marmorata Martin 1837), fishing cat (Prionailurus viverrinus Bennett 1833), Asian leopard cat (Prionailurus bengalensis Kerr 1792), flat-headed cat (Prionailurus planiceps Vigors and Horsfield 1872), jungle cat (Filis chaus Guldenstaedt 1776), Asiatic golden cat (Catopuma temminckii Vigors and Horsfield 1827), clouded leopard (Neofelis nebulosa Griffish 1821), leopard (Panthera pardus Linnaeus 1758) and tiger (Panthera tigris Linnaeus 1758) (Lekagul and McNeely 1977, 1988).

The Asian leopard cat is a small wild cat that is found in Southeast Asia. On average it is as large as a domestic cat, but there are considerable regional differences. The fur is also quite variable; it is yellow in the southern populations, but silver-grey in the northern ones. The chest and the lower part of the head are white. The Asian leopard cat bears black markings, that may be dependent on the subspecies spots or rosettes. It is a nocturnal animal and usually eats rodents, birds, fish,

*Corresponding author, e-mail: tanomtong@hotmail.com 
reptiles and small mammals. It is usually a solitary animal except for during the mating season. It has litters of 2 to 4 kittens and the gestation period can vary from 65 to $70 \mathrm{~d}$. The habitat of this cat is forests and rainforests both in low and mountainous areas, and usually not arid areas. It lives close to watercourses and may be found at heights of up to $3000 \mathrm{~m}$. The Asian leopard cat can climb trees skillfully. It is also able to swim, but will seldom do so. (Lekagul and McNeely 1977, 1988).

Although karyotypic studies of the family Felidae have been reported (Makino and Tateishi 1952, Thuline and Norby 1961, Hsu 1962, Hsu et al. 1963a, 1963b, Hsu and Rearden 1965, Ohno et al. 1962, Matano 1963, Chu et al. 1964, Benirschke and Low 1966, Leyhausen and Tonkin 1968, Sutton 1968, Hard 1968, Wurster and Benirschke1967, 1968a, 1968b, Wurster 1969, Milosevic et al. 1972, Wurster-Hill 1973, Wurster-Hill and Meritt 1974, Yang et al. 2000, Nie et al. 2002), there are few reports of the cytogenetics of the family Felidae in this species in Thailand. Animals in this family are conserved animals and are at risk. In this study, lymphocyte culture, conventional staining, G-banding and high-resolution technique were used to compare the results with the previous studies. In the future, basic knowledge about family Felidae and their cytogenetics will be applied for research and to protect them from extinction.

\section{Materials and methods}

Blood samples from the jugular vein were collected from 2 males and 2 females of the Asian leopard cat, which were kept in Nakhon Ratchasima Zoo, Nakhon Ratchasima province and Songkhla Zoo, Songkhla province, Thailand, using aseptic technique. The samples were kept in $10 \mathrm{ml}$ vacuum tubes containing heparin to prevent blood clotting and they were cooled on ice until arriving at the laboratory.

\section{Cell preparation}

The lymphocytes were cultured using the whole blood microculture technique adapted from Rooney (2001) and Kampiranont (2003).

Cell culture: The RPMI 1640 medium was prepared with 2\% PHA (phytohemagglutinin) as a mitogen and kept in blood culture bottles of $5 \mathrm{ml}$ each. A blood sample of $0.5 \mathrm{ml}$ was dropped into a medium bottle and well mixed. The culture bottle was loosely capped, incubated at $37^{\circ} \mathrm{C}$ under $5 \%$ carbon dioxide environment and regularly shaken in the morning and evening. When reaching harvest time at the $72^{\text {nd }}$ hour of incubation, colchicine was introduced and well mixed followed by further incubation for $30 \mathrm{~min}$.

Cell harvest: The blood sample mixture was centrifuged at 1,200 rpm for $10 \mathrm{~min}$ and the supernatant was discarded. $10 \mathrm{ml}$ of hypotonic solution $(0.075 \mathrm{M} \mathrm{KCl})$ was applied to the pellet and the mixture was incubated for $30 \mathrm{~min}$. $\mathrm{KCl}$ was discarded with the supernatant after centrifugation again at 1,200 rpm for $10 \mathrm{~min}$. Cells were fixed by fresh cool fixative (methanol:glacial acetic acid $=3: 1$ ) gradually added up to $8 \mathrm{ml}$ before centrifuging again at 1,200 rpm for $10 \mathrm{~min}$ and the supernatant discarded. The fixation was repeated until the supernatant was clear and the pellet was mixed with $1 \mathrm{ml}$ fixative. The mixture was dropped onto a clean and cold slide using micropipette followed by the air-dry technique. The materials or slide was conventionally stained with $20 \%$ stock Giemsa's solution for $30 \mathrm{~min}$.

\section{G-banding method}

The G-banding technique was adapted from Kampiranont (2003). The slide was well dried and then soaked in working trypsin $(0.025 \%$ trypsin EDTA $)$ at $37^{\circ} \mathrm{C}$ before the termination of trypsin activity by washing the slide with $10 \%$ fetal calf serum (FCS) or phosphate buffer. FCS was washed out by $50 \%$ methanol and finaly materials or the slide was stained with $10 \%$ Giemsa's solution for 

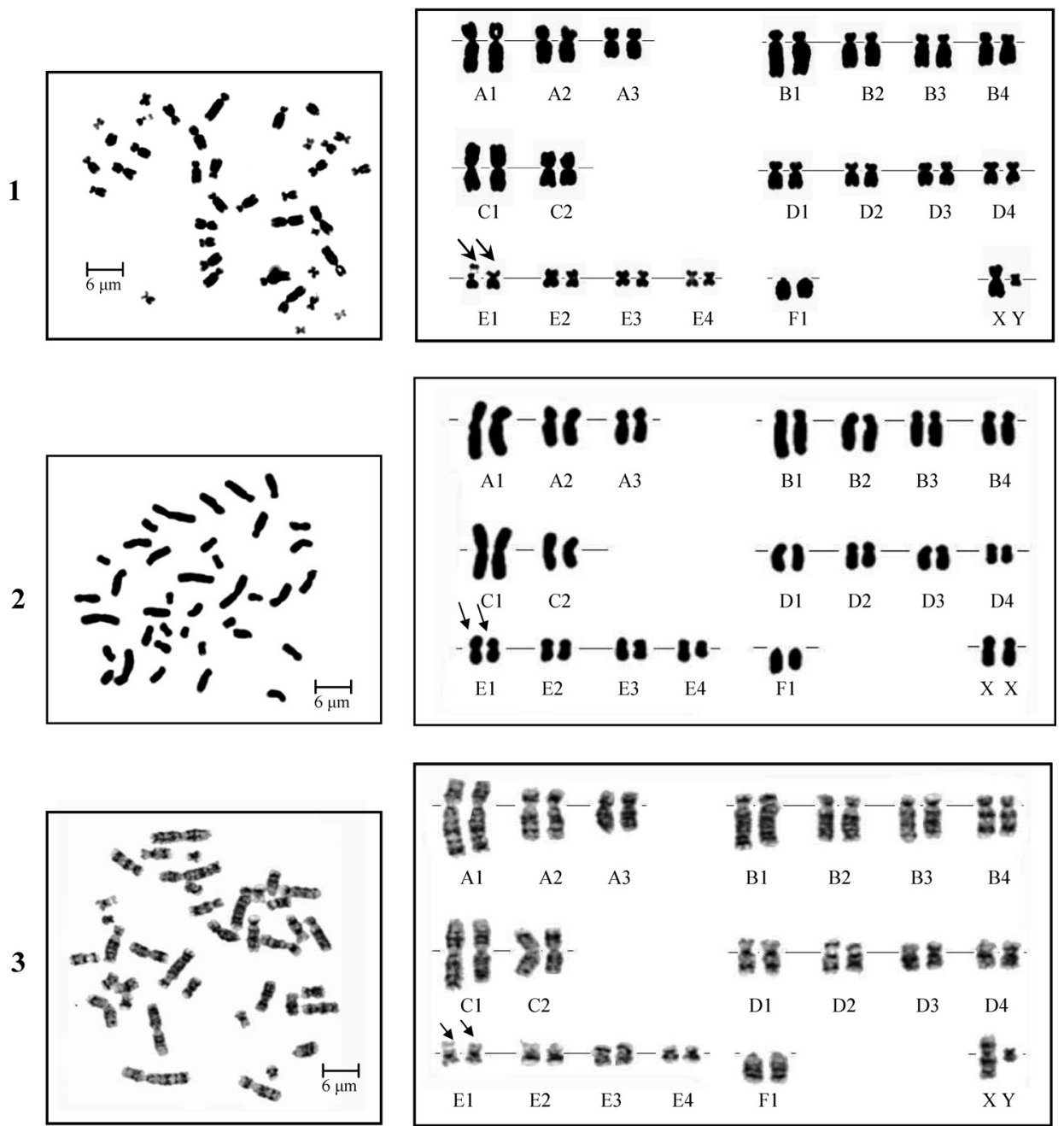

Fig. 1-3. Metaphase chromosome and karyotype of a male Asian leopard cat (Prionailurus bengalensis) $2 n$ (diploid) $=38$, by conventional staining, showing satellite chromosomes (arrows).

30 min.

\section{High-resolution staining method}

After the lymphocytes were cultured for $72 \mathrm{~h}, 0.05 \mathrm{ml}$ of $10^{-5} \mathrm{M}$ methotrexate was applied into the cultured lymphocytes to induce synchronization. The mixture was incubated again for $17 \mathrm{~h}$ before the methotrexate was discarded with the supernatant by centrifugution at $2,800 \mathrm{rpm}$. The pellet was mixed with $5 \mathrm{ml}$ of the RPMI 1640 medium and centrifuged at 2,800 rpm. The supernatant was discarded before the cultured cells were released by adding $0.2 \mathrm{ml}$ thymidine and incubating for $5 \mathrm{~h}$ and $15 \mathrm{~min}$. The cells were harvested at the exact time and stained using the G-banding procedure.

\section{Chromosomal checks, karyotyping and idiograming}

Chromosomal checks were performed on mitotic metaphase cells under a light microscope. Twenty cells each of male and female with clearly observable and well-spread chromosomes were selected and photographed. The length short arm chromosome (Ls) and the length long arm chro- 

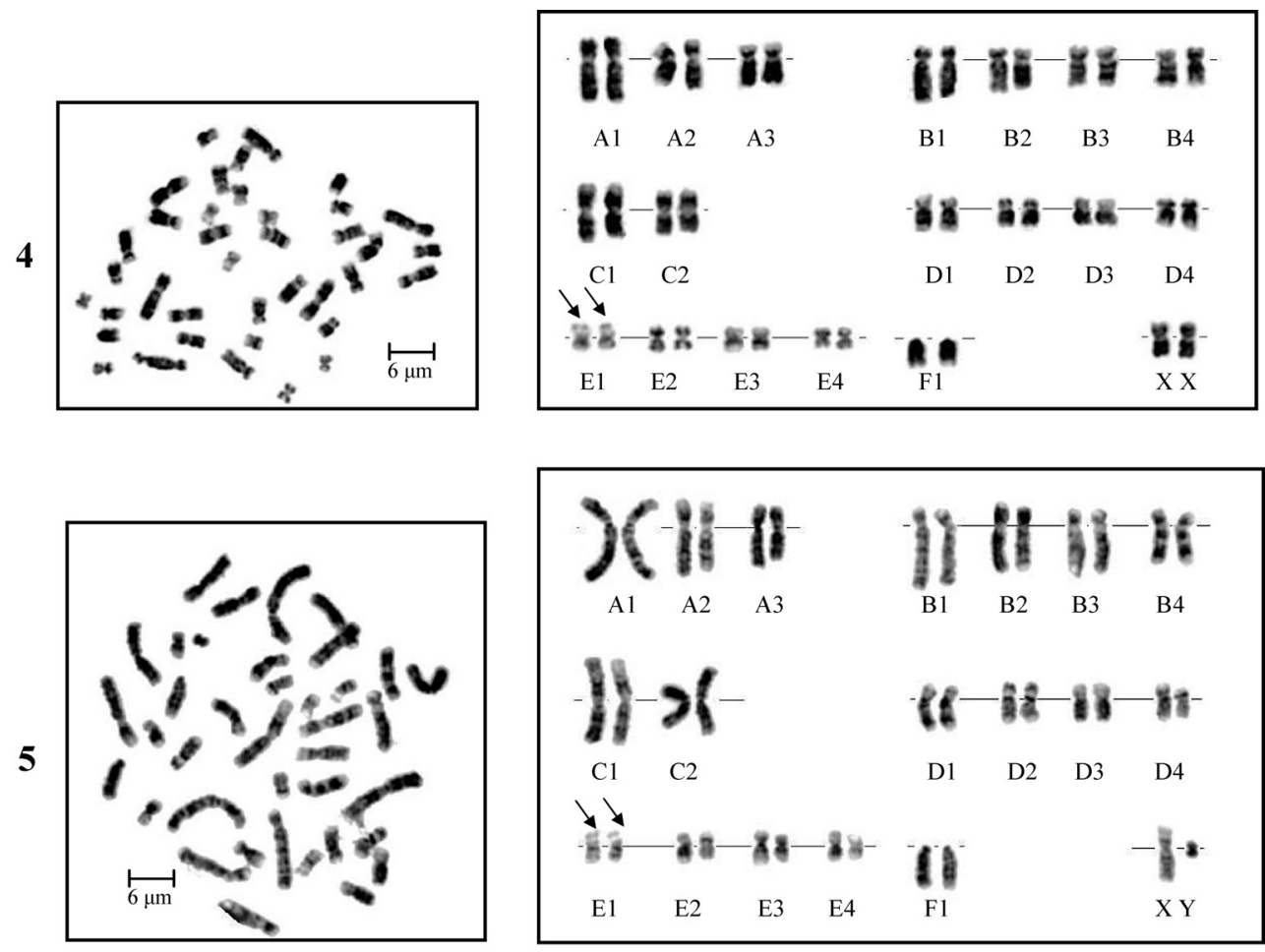
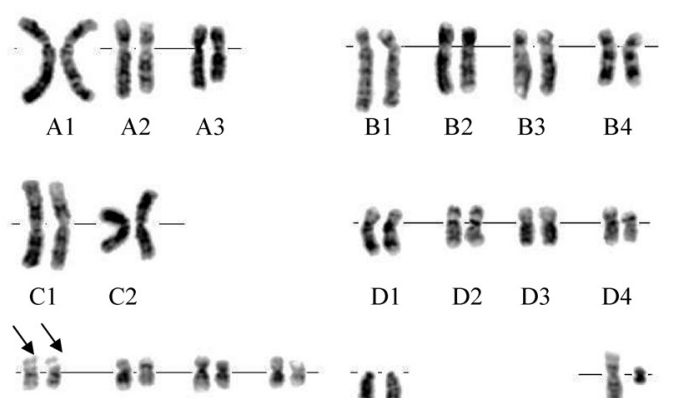

E1 E2 E3 E4
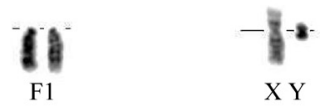

Fig. 4-6. Metaphase chromosome and karyotype of a female Asian leopard cat (Prionailurus bengalensis) $2 n$ (diploid) $=38$, by G-banding, showing satellite chromosomes (arrows).

mosome $(\mathrm{Ll})$ were measured to calculate the total length arm chromosome (LT, $\mathrm{LT}=\mathrm{Ls}+\mathrm{Ll})$. The relative length (RL), the centromeric index (CI) and standard deviation (SD) of RL, CI were also computed to classify the types of chromosomes according to Chaiyasut (1989). All parameters were used in karyotyping and idiograming according to Hsu and Rearden (1965).

\section{Results}

Karyotypic study of the Asian leopard cat using lymphocyte culture revealed that the chromosome number is $2 n$ (diploid) $=38$, and the fundamental number (NF) is 74 in male and female. The autosomes of the Asian leopard cat composed of 6 types: A type had 4 large submetacentric, 2 medium submetacentric chromosomes, B type had 6 large acrocentric, 2 medium acrocentric chro- 


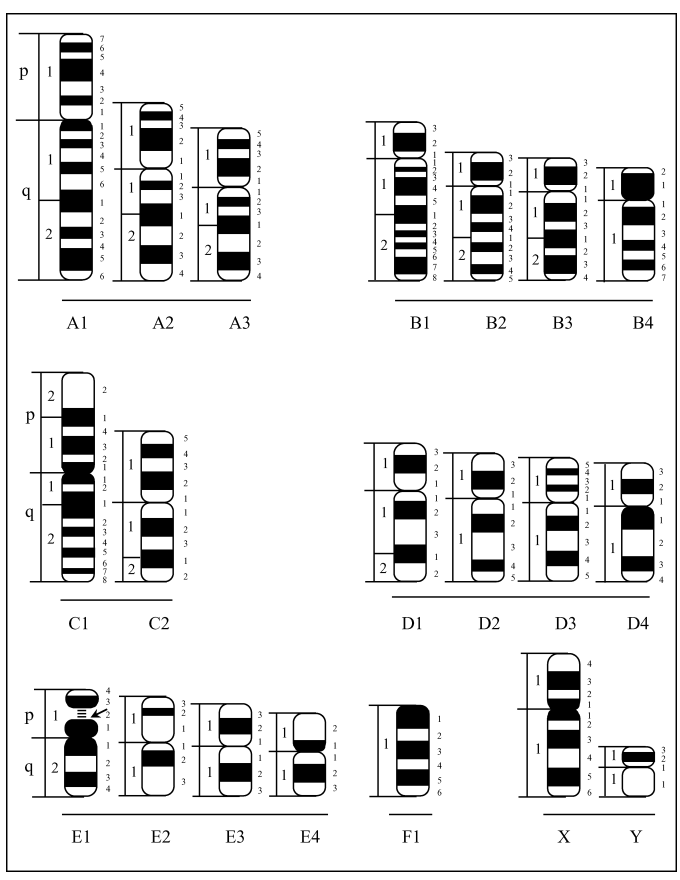

Fig. 7. Idiogram of the Asian leopard cat (Prionailurus bengalensis) $2 n$ (diploid) $=38$, by G-banding, showing satellite chromosome (arrow).

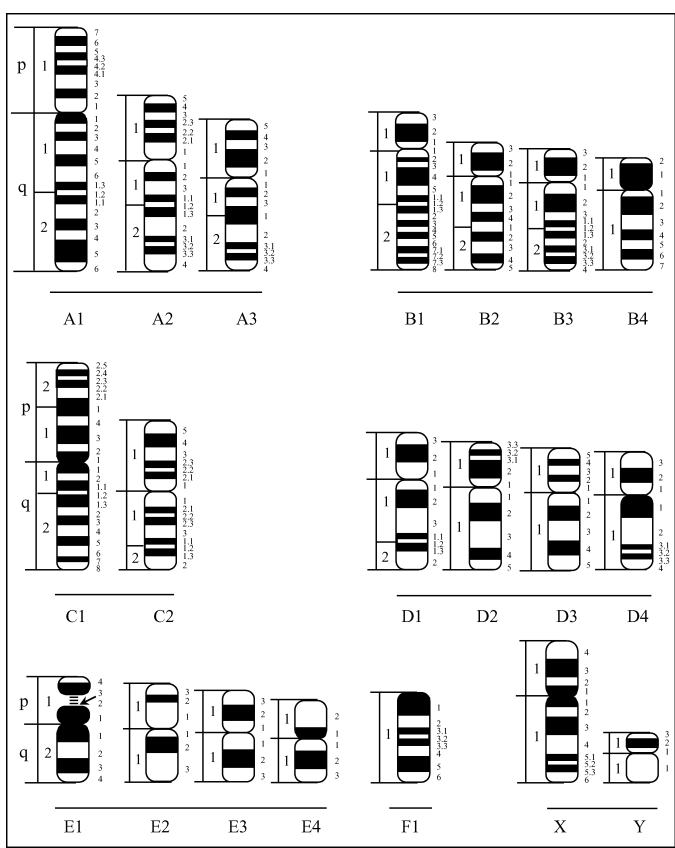

Fig. 8. Idiogram of the Asian leopard cat (Prionailurus bengalensis) $2 n$ (diploid) $=38$, by high-resolution technique, showing satellite chromosome (arrow).

mosomes, C type had 4 large metacentric chromosomes, D type had 8 small submetacentric chromosomes, E type had 8 small metacentric chromosomes and $\mathrm{F}$ type had 2 small telocentric chromosomes. In addition, a pair of the short arm of chromosome E1 (chromosome pairs 14) showed a clearly observable satellite chromosomes. The X-chromosome was medium submetacentric chromosome and the Y chromosome was the smallest metacentric chromosome (Figs. 1-6).

The important chromosome marker of the Asian leopard cat is the asymmetrical karyotype, in which all 4 types of chromosomes are found (metacentric, submetacentric, telocentric and acrocentric). The largest and smallest chromosomes show large size difference (approximately 5.2 fold). The largest chromosome is submetacentric chromosome, while the second largest chromosome is acrocentric chromosome and the $\mathrm{Y}$ chromosome is the smallest metacentric chromosome (Figs. 1-6). The G-banding revealed that the number of G-bands on one set of haploid chromosomes, which includes autosomes, $\mathrm{X}$ and $\mathrm{Y}$ chromosomes, is 183 bands for the Asian leopard cat. The number of bands in one set of prometaphase haploid chromosomes from the high-resolution method is 236 bands (Figs. 7, 8).

Comparison of chromosome banding pattern between the Asian leopard cat and the domestic cat (Felis catus) revealed that 13 chromosome pairs show the same pattern (pairs A1, A2, A3, B3, B4, C1, C2, D3, D4, E1, E3, F1 and X chromosome) and 4 chromosome pairs share similarities (pairs B1, B2, D2 and E2) (Fig. 9). This indicates that there is an evolutionary relationship between the Asian leopard cat and the domestic cat. The data of the chromosomal checks on mitotic metaphase cells of the Asian leopard cat are shown in Tables 1 and 2. Fig. 7 shows the idiogram for the Asian leopard cat from the G-band staining, while Fig. 8 shows the idiogram from the high-resolution banding with landmarks, bands and sub-bands. The karyotype formula for the Asian leopard cat are as follows: 
Table 1. Mean of length, short arm chromosome (Ls); length, long arm chromosome (L1); length, total arm chromosome (LT); relative length (RL); centromeric index (CI) and standard deviation (SD) of RL from metaphase chromosome of 10 cells in male Asian leopard cat (Prionallurus bengalensis) $2 n=38$

\begin{tabular}{cccccccc}
\hline \hline $\begin{array}{c}\text { Chromosome } \\
\text { pairs }\end{array}$ & Ls & Ll & LT & CI \pm SD & RL \pm SD & $\begin{array}{c}\text { Size of } \\
\text { Chromosome }\end{array}$ & $\begin{array}{c}\text { Type of } \\
\text { Chromosome }\end{array}$ \\
\hline A1 & 0.760 & 1.432 & 2.192 & $0.648 \pm 0.023$ & $0.049 \pm 0.004$ & $\mathrm{sm}$ & $\mathrm{L}$ \\
A2 & 0.390 & 0.964 & 1.354 & $0.638 \pm 0.028$ & $0.034 \pm 0.003$ & $\mathrm{sm}$ & $\mathrm{L}$ \\
A3 & 0.455 & 0.845 & 1.300 & $0.638 \pm 0.047$ & $0.029 \pm 0.003$ & $\mathrm{sm}$ & $\mathrm{M}$ \\
B1 & 0.375 & 1.462 & 1.837 & $0.789 \pm 0.023$ & $0.041 \pm 0.002$ & $\mathrm{a}$ & $\mathrm{L}$ \\
B2 & 0.375 & 1.114 & 1.489 & $0.750 \pm 0.025$ & $0.033 \pm 0.002$ & $\mathrm{a}$ & $\mathrm{L}$ \\
B3 & 0.335 & 1.056 & 1.391 & $0.755 \pm 0.038$ & $0.031 \pm 0.002$ & $\mathrm{a}$ & $\mathrm{L}$ \\
B4 & 0.335 & 0.957 & 1.292 & $0.732 \pm 0.034$ & $0.029 \pm 0.003$ & $\mathrm{a}$ & $\mathrm{M}$ \\
C1 & 0.835 & 1.040 & 1.875 & $0.525 \pm 0.014$ & $0.044 \pm 0.003$ & $\mathrm{~m}$ & $\mathrm{~L}$ \\
C2 & 0.620 & 0.752 & 1.372 & $0.533 \pm 0.018$ & $0.031 \pm 0.003$ & $\mathrm{~m}$ & $\mathrm{~L}$ \\
D1 & 0.285 & 0.674 & 0.959 & $0.662 \pm 0.057$ & $0.023 \pm 0.003$ & $\mathrm{sm}$ & $\mathrm{S}$ \\
D2 & 0.255 & 0.628 & 0.883 & $0.667 \pm 0.043$ & $0.021 \pm 0.002$ & $\mathrm{sm}$ & $\mathrm{S}$ \\
D3 & 0.285 & 0.592 & 0.877 & $0.648 \pm 0.040$ & $0.020 \pm 0.002$ & $\mathrm{sm}$ & $\mathrm{S}$ \\
D4 & 0.270 & 0.589 & 0.859 & $0.663 \pm 0.044$ & $0.020 \pm 0.003$ & $\mathrm{sm}$ & $\mathrm{S}$ \\
E1 & 0.350 & 0.405 & 0.755 & $0.537 \pm 0.038$ & $0.017 \pm 0.002$ & $\mathrm{~m}$ & $\mathrm{~S}$ \\
E2 & 0.320 & 0.370 & 0.690 & $0.542 \pm 0.028$ & $0.015 \pm 0.001$ & $\mathrm{~m}$ & $\mathrm{~S}$ \\
E3 & 0.220 & 0.341 & 0.561 & $0.537 \pm 0.025$ & $0.014 \pm 0.002$ & $\mathrm{~m}$ & $\mathrm{~S}$ \\
E4 & 0.215 & 0.303 & 0.518 & $0.536 \pm 0.034$ & $0.013 \pm 0.002$ & $\mathrm{~m}$ & $\mathrm{~S}$ \\
F1 & 0.000 & 0.841 & 0.841 & $1.000 \pm 0.000$ & $0.019 \pm 0.004$ & $\mathrm{t}$ & $\mathrm{S}$ \\
X & 0.460 & 0.696 & 1.156 & $0.609 \pm 0.057$ & $0.026 \pm 0.003$ & $\mathrm{sm}$ & $\mathrm{M}$ \\
Y & 0.160 & 0.234 & 0.394 & $0.595 \pm 0.044$ & $0.009 \pm 0.001$ & $\mathrm{~m}$ & $\mathrm{~S}$ \\
\hline
\end{tabular}

Remark: $\mathrm{L}=$ large chromosome $(\mathrm{LT}>1.320), \mathrm{M}=$ medium chromosome $(\mathrm{LT}=1.027-1.320), \mathrm{S}=$ small chromosome $(\mathrm{LT}<1.027), \mathrm{m}=$ metacentric chromosome, $\mathrm{sm}=$ submetacentric chromosome, $\mathrm{a}=$ acrocentric chromosome, $\mathrm{t}=$ telocentric chromosome.

\section{$2 n(38)=\mathrm{L}_{4}{ }^{\mathrm{m}}+\mathrm{L}_{4}{ }^{\mathrm{sm}}+\mathrm{L}_{6}{ }^{\mathrm{a}}+\mathrm{M}_{2}{ }^{\mathrm{sm}}+\mathrm{M}_{2}{ }^{\mathrm{a}}+\mathrm{S}_{8}{ }^{\mathrm{m}}+\mathrm{S}_{8}{ }^{\mathrm{sm}}+\mathrm{S}_{\mathrm{t}}{ }^{2}+$ Sex-chromosome}

\section{Discussion}

Karyotypic study of the Asian leopard cat using lymphocyte culture revealed that the chromosome number is $2 n=38$. This result agrees with the previous studies by Makino and Tateishi (1952) indicating that a member of the Asian leopard cat family has $2 n=38$. This corresponds to $2 n$ for the members of the family Felidae according to reports in puma (Felis concolor), Canadian lynx ( $F$. lynx), snow leopard (Uncia uncia), jaguarundi (F. yagouaroundi), cheetah (Acinonyx jabatus jabatus), serval (F. serval), fishing cat ( $F$. viverrina), Bob cat (Lynx rufus), marbled cat (F. marmora$t a$ ), European wild cat (F. silvestris), black footed cat (F. nigripes), leopard (Panthera pardus), tiger (P. tigris), domestic cat (F. catus) and ocelot (F. pardalis) (Makino and Tateishi 1952, Thuline and Norby 1961, Hsu 1962, Hsu et al. 1963a, 1963b, Hsu and Rearden 1965, Ohno et al. 1962, Matano 1963, Chu et al. 1964, Benirschke and Low 1966, Leyhausen and Tonkin 1968, Sutton 1968, Hard 1968, Wurster and Benirschke 1967, 1968a, 1968b, Wurster 1969, Milosevic et al. 1972, WursterHill 1973, Wurster-Hill and Meritt 1974). However this number differs from the chromosome number of Geoffroy's (F. geoffroyi) and Marguay (F. wiedi) which is $2 n=36$ (Hsu 1962, Hsu et al. 1963).

The autosomes of the Asian leopard cat can be separated to 6 types: A type had 4 large submetacentric and 2 medium submetacentric chromosomes, B type had 6 large acrocentric and 2 medium acrocentric chromosomes, $\mathrm{C}$ type had 4 large metacentric chromosomes, D type had 8 
Table 2. Mean of length, short arm chromosome (Ls); length, long arm chromosome (L1); length, total arm chromosome (LT); relative length (RL); centromeric index (CI) and standard deviation (SD) of RL from metaphase chromosome of 10 cells in female Asian leopard cat (Prionallurus bengalensis) $2 n=38$

\begin{tabular}{cccccccc}
\hline \hline $\begin{array}{c}\text { Chromosome } \\
\text { pairs }\end{array}$ & Ls & L1 & LT & CI \pm SD & RL \pm SD & $\begin{array}{c}\text { Size of } \\
\text { Chromosome }\end{array}$ & $\begin{array}{c}\text { Type of } \\
\text { Chromosome }\end{array}$ \\
\hline A1 & 0.760 & 1.432 & 2.192 & $0.648 \pm 0.023$ & $0.049 \pm 0.004$ & sm & $\mathrm{L}$ \\
A1 & 0.716 & 1.338 & 2.054 & $0.651 \pm 0.022$ & $0.045 \pm 0.004$ & sm & $\mathrm{L}$ \\
A2 & 0.536 & 0.936 & 1.472 & $0.636 \pm 0.026$ & $0.032 \pm 0.002$ & sm & $\mathrm{L}$ \\
A3 & 0.481 & 0.802 & 1.283 & $0.625 \pm 0.044$ & $0.028 \pm 0.002$ & $\mathrm{sm}$ & $\mathrm{M}$ \\
B1 & 0.398 & 1.364 & 1.761 & $0.774 \pm 0.029$ & $0.039 \pm 0.002$ & $\mathrm{a}$ & $\mathrm{L}$ \\
B2 & 0.372 & 1.054 & 1.426 & $0.739 \pm 0.028$ & $0.031 \pm 0.002$ & $\mathrm{a}$ & $\mathrm{L}$ \\
B3 & 0.354 & 0.988 & 1.342 & $0.737 \pm 0.0410$ & $0.030 \pm 0.001$ & $\mathrm{a}$ & $\mathrm{L}$ \\
B4 & 0.359 & 0.907 & 1.266 & $0.716 \pm 0.037$ & $0.028 \pm 0.002$ & $\mathrm{a}$ & $\mathrm{M}$ \\
C1 & 0.907 & 0.984 & 1.891 & $0.520 \pm 0.013$ & $0.042 \pm 0.003$ & $\mathrm{~m}$ & $\mathrm{~L}$ \\
C2 & 0.643 & 0.719 & 1.362 & $0.528 \pm 0.015$ & $0.030 \pm 0.002$ & $\mathrm{~m}$ & $\mathrm{~L}$ \\
D1 & 0.355 & 0.662 & 1.017 & $0.651 \pm 0.051$ & $0.022 \pm 0.002$ & $\mathrm{sm}$ & $\mathrm{S}$ \\
D2 & 0.331 & 0.599 & 0.930 & $0.644 \pm 0.047$ & $0.020 \pm 0.001$ & $\mathrm{sm}$ & $\mathrm{S}$ \\
D3 & 0.331 & 0.570 & 0.900 & $0.633 \pm 0.040$ & $0.020 \pm 0.001$ & $\mathrm{sm}$ & $\mathrm{S}$ \\
D4 & 0.317 & 0.549 & 0.866 & $0.634 \pm 0.053$ & $0.019 \pm 0.002$ & $\mathrm{sm}$ & $\mathrm{S}$ \\
E1 & 0.375 & 0.418 & 0.792 & $0.527 \pm 0.036$ & $0.017 \pm 0.002$ & $\mathrm{~m}$ & $\mathrm{~S}$ \\
E2 & 0.327 & 0.383 & 0.710 & $0.539 \pm 0.035$ & $0.016 \pm 0.002$ & $\mathrm{~m}$ & $\mathrm{~S}$ \\
E3 & 0.308 & 0.354 & 0.662 & $0.535 \pm 0.023$ & $0.015 \pm 0.001$ & $\mathrm{~m}$ & $\mathrm{~S}$ \\
E4 & 0.273 & 0.313 & 0.586 & $0.535 \pm 0.029$ & $0.013 \pm 0.001$ & $\mathrm{~m}$ & $\mathrm{~S}$ \\
F1 & 0.000 & 0.855 & 0.855 & $1.000 \pm 0.00$ & $0.019 \pm 0.003$ & $\mathrm{t}$ & $\mathrm{S}$ \\
X & 0.448 & 0.681 & 1.129 & $0.603 \pm 0.043$ & $0.025 \pm 0.007$ & $\mathrm{sm}$ & $\mathrm{M}$ \\
Y & 0.160 & 0.234 & 0.394 & $0.594 \pm 0.044$ & $0.009 \pm 0.001$ & $\mathrm{~m}$ & $\mathrm{~S}$ \\
\hline
\end{tabular}

Remark: $\mathrm{L}=$ large chromosome $(\mathrm{LT}>1.320), \mathrm{M}=$ medium chromosome $(\mathrm{LT}=1.027-1.320), \mathrm{S}=$ small chromosome $(\mathrm{LT}<1.027), \mathrm{m}=$ metacentric chromosome, $\mathrm{sm}=$ submetacentric chromosome, $\mathrm{a}=$ acrocentric chromosome, $\mathrm{t}=$ telocentric chromosome.

small submetacentric chromosomes, E type had 8 small metacentric chromosomes and F type had 2 small telocentric chromosomes. This result agrees with the previous studies by Makino and Tateishi (1952) indicating that a member of the Asian leopard cat family had 6 types of autosome: A type had 6 submetacentric chromosomes, B type had 8 acrocentric chromosomes, $\mathrm{C}$ type had 4 metacentric chromosomes, D type had 8 submetacentric chromosomes, E type had 8 metacentric chromosomes and $\mathrm{F}$ type had 2 telocentric chromosomes. The comparison of the Asian leopard cat chromosomes with the domestic cat revealed that there is a difference in chromosomes of $\mathrm{E}$ and $\mathrm{F}$ type. The Asian leopard cat had 4 and 1 pairs of chromosomes whereas the domestic cat had 3 and 2 pairs of chromosomes, respectively.

The fundamental number (NF) of the Asian leopard cat is 74 in male and female. For the sex chromosomes, the $\mathrm{X}$-chromosome was medium submetacentric chromosome and $\mathrm{Y}$ chromosome was the smallest metacentric chromosome. This result was different from Makino and Tateishi (1952) who reported that the X and Y chromosomes of the Asian leopard cat were submetacentric chromosomes. The comparison of the Asian leopard cat X and Y chromosomes to the domestic cat indicated that $\mathrm{X}$ chromosome was the medium submetacentric and $\mathrm{Y}$ chromosome was the smallest subtelocentric (Thuline and Norby 1961, Ohno et al. 1962, Matano 1963, Chu et al. 1964, Hsu and Rearden 1965). The comparison of the Asian leopard cat X and Y chromosomes to animals in family Felidae in Thailand namely, leopard (Panthera pardus) and clouded leopard (Pardofelis nebulosa) found that $\mathrm{X}$ and $\mathrm{Y}$ chromosomes were submetacentric in every species (Fig. 10).

From this study, the Asian leopard cat had the chromosome marker at the short arm of chro- 


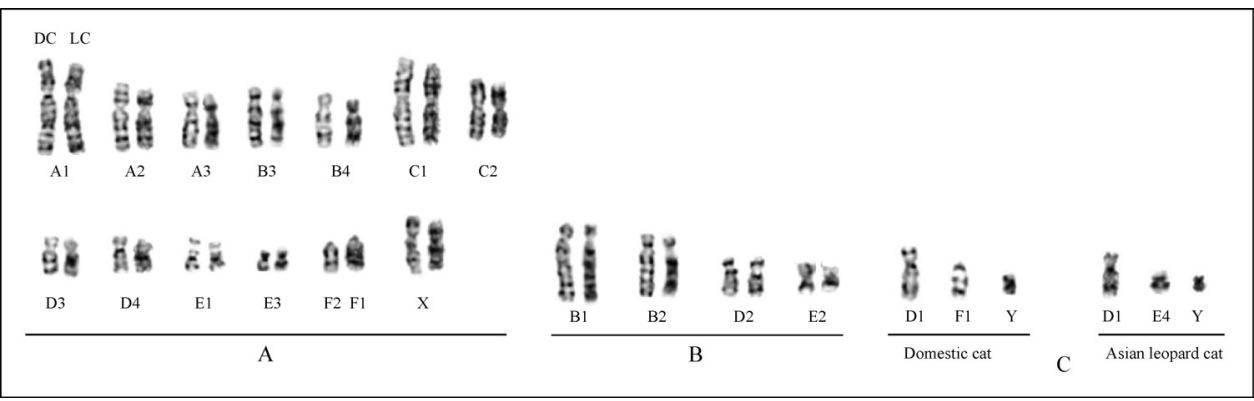

Fig. 9. A comparison of the chromosome of animal species in the family Felidae in Thailand, between domestic cat, Felis catus (DC, left) (Nie et al. 2002) and Asian leopard cat, Prionailurus bengalensis (LC, right) in this study, show the same (A), similar (B) and different (C) by G-banding patterns.

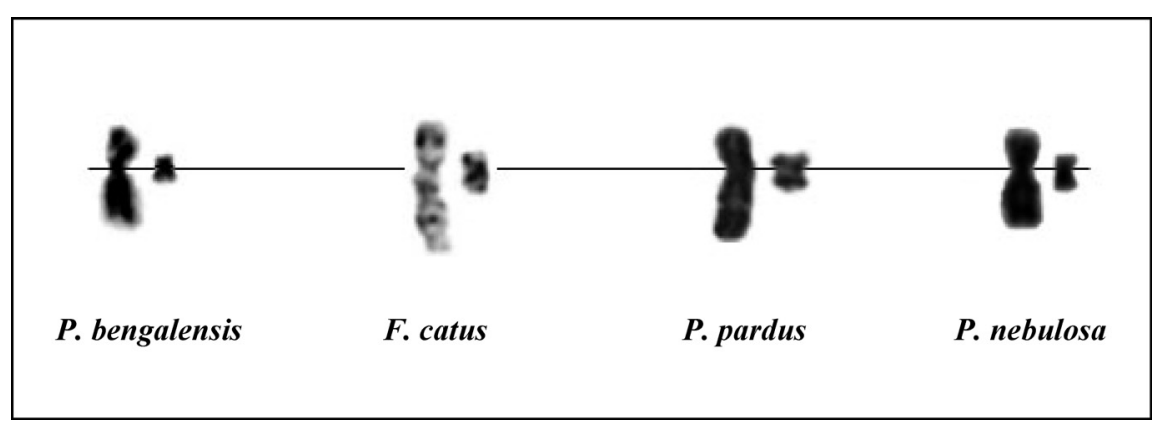

Fig. 10. A comparison of the sex-chromosome of animal species in the family Felidae in Thailand: Asian leopard cat (Prionailurus bengalensis), domestic cat (Felis catus), leopard (Panthera pardus) and clouded leopard (Pardofelis nebulosa).

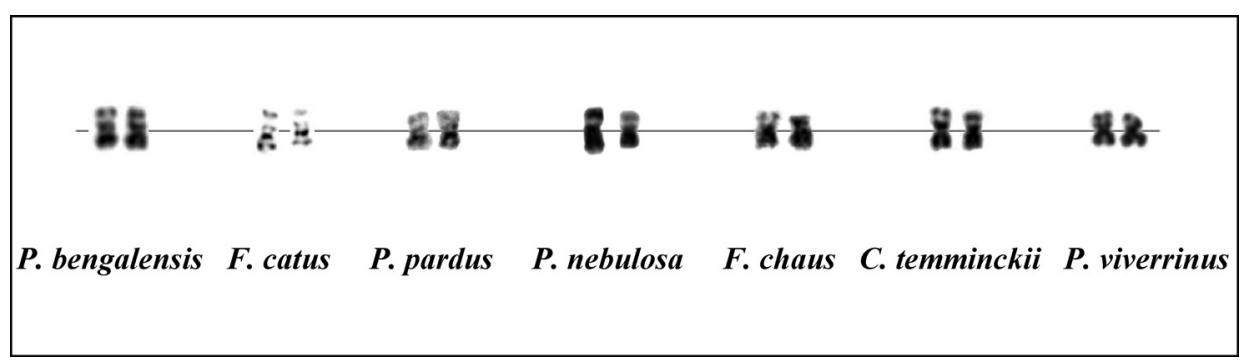

Fig. 11. A comparison of the satellite chromosome (nucleolar organizer regions, NOR) of animal species in the family Felidae in Thailand: Asian leopard cat (Prionailurus bengalensis), domestic cat (Felis catus), leopard (Panthera pardus), clouded leopard (Pardofelis nebulosa), jungle cat (Felis chaus), Asian golden cat (Catopuma temminckii) and fishing cat (Prionailurus viverrinus).

mosomes E1 (chromosome pairs 14) that was a satellite chromosomes with nucleolar organizer regions, NOR. This result agrees with previous reports about the chromosomes E1 of animals in the family Felidae having a satellite chromosomes (Makino and Tateishi 1952, Thuline and Norby 1961, Hsu 1962, Hsu et al. 1963a, 1963b, Hsu and Rearden 1965, Ohno et al. 1962, Matano 1963, Chu et al. 1964, Benirschke and Low 1966, Leyhausen and Tonkin 1968, Sutton 1968, Hard 1968, Wurster and Benirschke 1967, 1968a, 1968b, Wurster 1969, Milosevic et al. 1972, Wurster-Hill 1973, Wurster-Hill and Meritt 1974) (Fig. 11). 
The G-banding revealed that the number of G-bands on 1 set of haploid chromosomes, which includes autosomes, $\mathrm{X}$ and $\mathrm{Y}$ chromosomes, is 183 bands for the Asian leopard cat. The number of bands in 1 set of prometaphase haploid chromosomes from the high-resolution method is 236 bands. Comparing with the study in a domestic cat by Yang et al. (2000), there are 317 bands on 1 set of the metaphase haploid chromosomes. A recent study showed a lower number of bands compared to previous studies because only clearly observable bands of the chromosomes were counted.

Comparison of chromosome banding pattern between the Asian leopard cat and the domestic cat (Nie et al. 2002) revealed that 13 chromosome pairs show the same pattern (pairs A1, A2, A3, B3, B4, C1, C2, D3, D4, E1, E3, F1 and X chromosome) and 4 pairs share similarities (pairs B1, $\mathrm{B} 2, \mathrm{D} 2$ and E2). This indicates that there is evolutionary relationship between the Asian leopard cat and the domestic cat. For further studies, more information about genetic differences is needed which may be accomplished by using molecular biology or molecular genetics.

\section{Acknowledgement}

The financial support from The Zoological Park Organization Under the Royal Patronage of H.M. The King is gratefully acknowledged. We also thank Mr. Sopon Dumnui, Director of the Organization and Dr. Sumat Kamolnaranath, chief of the Educational Division, for valuable help. We would like to thank the Directors of the Nakhon Ratchasima Zoo and Songkhla Zoo for the blood samples. Thanks to the authorities and officers of these zoos for good cooperation.

\section{References}

Benirschke, K. and Low, R. J. 1966. Chromosome studies of four carnivores. Mammalian Chromosome Newsletter 21: $148-160$.

Chaiyasut, K. 1989. Cytogenetics and cytotaxonomy of the family Zephyranthes. Department of Botany, Faculty of Science, Chulalongkorn University, Bangkok, Thailand.

Chu, E. H. Y., Thuline, H. C. and Norby, D. E. 1964. Triploid-diploid chimerism in a male tortoiseshell cat. Cytogenetics 3: $1-8$.

Hard, W. L. 1968. The karyotype of a male cheetah, Acinonyx jabatus jabatus. Mamm. Chrom. Newsletter 9: 16-25.

Hsu, T. C. 1962. Two species of cats with 36 chromosomes. Mamm. Chrom. Newsletter 8: 4-10.

-, Rearden, H. H. and Luquette, G. F. 1963a. Karyological studies of nine species of Felidae. America Naturalist 97: $225-235$.

-, Arrighi, F. E. and Luquette, G. F. 1963b. Karyological studies of nine species of Felidae. America Naturalist 97: $225-232$.

— and Rearden, H. H. 1965. Further karyological studies on Felidae. Chromosoma (Berl.) 16: 365-371.

Kampiranont, A. 2003. Cytogenetics. Department of Genetics, Faculty of Science, Kasetsart University, Bangkok, Thailand.

Lekagul, B. and McNeely, J. A. 1977. Mammals of Thailand. $1^{\text {st }}$ ed. Kurusapha Ladprao Press: Bangkok, Thailand.

- and - 1988. Mammals of Thailand. $2^{\text {nd }}$ ed. Sahakarn Bhaet: Bangkok, Thailand.

Leyhausen, P. and Tonkin, B. A. 1968. Comment on the karyotypes of the leopard cat and fishing cat. Mamm. Chrom. Newsletter 9: 78-85.

Makino, S. and Tateishi, S. 1952. A comparison of the chromosomes of the lion, Chinese leopard cat and house cat. J. Morphology 90: 93-99.

Matano, Y. 1963. A study of the chromosome in the cat. Japan J. Gene. 38: 147-152.

Milosevic, M., Zivkovic, S. and Isakovic, I. 1972. Hromozomske karakteristike nekin carnivora Iz Srbij. Genetika (Beogard) 4: 1-9.

Nie, W., Wang, J., O’Brien, P. C. M., Fu, B. and Ying, T. 2002. The genome phylogeny of domestic cat, red panda and mustelid species revealed by comparative chromosome painting and G-banding. Chrom. Res. 10: 209-222.

Ohno, S., Stenius, C., Weiler, C. P., Trujillo, J. M., Kaplan, W. D. and Kinosita, R. 1962. Early meiosis of male germ cells in fetal testis of Felis domestica. Exp. Cell Res. 27: 401-410.

Rooney, D. E. 2001. Human Cytogenetics: Constitutional Analysis. Oxford University Press: Oxford.

Sutton, D. A. 1968. Karyotype of a female bobcat, Lynx rufus californicus. Mamm. Chrom. Newsletter 9: $249-254$.

Thuline, H. C. and Norby, D. E. 1961. Spontaneous occurrence of chromosome abnormality in cats. Science 134: $544-546$.

Wilson, D. E. and Cole, F. R. 2000. Common names of mammals of the world. Smithsonian Institution: United States of 
America.

Wurster, D. H. and Benirschke, K. 1967. Chromosome numbers in thirty species of Carnivora. Mamm. Chrom. Newsletter 8: $195-216$.

— and - 1968a. Comparative cytogenetic studies in the order Carnivora. Chromosoma 24: 336-382.

— and - 1968b. Karyotype of four more species of cats. Mamm. Chrom. Newsletter 9: 236-245.

- 1969. Cytogenetic and phylogenetic studies in Canivora. In: Comparative Mammalian Cytogenetics Benirschke, K., ed., Springger-Verlag, N.Y.

Wurster-Hill, D.H. 1973. Chromosome of eight species from five families of Carnivora. J. Mammalian 54: 763-782.

— and Meritt, D. A., Jr. 1974. The G-band chromosomes of the marbled cat, Felis marmorata. Mamm. Chrom. Newsletter 15: $14-19$.

Yang, F., Graphodatsky, A. S., O’Brien, P. C. M., Colabella, A., Solanky, N., Squire, M., Sargan, D. R. and Ferguson-Smith, M. A. 2000. Reciprocal chromosome painting illuminates the history of genome evolution of the domestic cat, dog and human. Chrom. Res. 8: 393-404. 